\title{
One-stage, Full-mouth Sulcular Debridement with FRP 1064 Nd:YAG as Adjunctive to a Full Mouth Initial Periodontal Therapy: A Clinical Case Series Retrospective Study
}

\section{Beatrijs Deruyter*}

Liedekerke, Belgium

*Corresponding Author: Beatrijs Deruyter, Liedekerke, Belgium
Received: September 25, 2021

Published: December 16, 2021

(C) All rights are reserved by Beatrijs

Deruyter.

\section{Abstract}

Objective: In an attempt to enhance the treatment outcomes of anti-infective periodontal therapy, the one-stage, full-mouth therapy has been the most logic way of treating chronic adult periodontitis. Still, quadrant scaling and root planing are considered standard treatment with accepted limited results and loss of dental structures. One-stage, full-mouth debridement with FRP 1064nm Nd:YAG in conjunction with ultrasonic scaling can provide a cost-effective alternative procedure for gain in clinical attachment to meet patients' and clinicians' expectations. In this limited study in a private practice, clinical attachment levels are compared to what is known as the standard conventional treatment in periodontics. One-stage, full-mouth procedure eliminates cross-contamination. The Thermal laser effect, might play an important role in the significant difference in results between compared to other alternative protocols to treat chronic periodontitis. Simplification of the protocol with FRP 1064nm Nd:YAG leads to a cost-effective procedure with more predictable results.

Summary Background Data: This retrospective study evaluates a series of clinical cases.

Material and Methods: Data were collected from 53 patients with a severe periodontal screening index. All were treated with the same protocol of one-stage, full-mouth disinfection of scaling, in combination with laser attachment procedure (LAP), FRP 1064 nm $\mathrm{Nd}$ :YAG and indicated antimicrobial therapy where necessary. Clinical attachment gain was compared to the results of other onestage, full-mouth procedures and quadrant treatment. Clinical evaluation of the attachment gain was assessed after two months, eight months, up to 14 months.

Conclusion: The combination of one-stage, full-mouth scaling, laser attachment procedure for debridement of the pocket, disinfection of the exposed dentine and specific indicated antibiotics where needed, provides a predictable outcome of statistically significant gain in attachment level. Because of its simplicity, the treatment can be performed in a one-hour chairside protocol and can be provided for most patients, even those with serious medical antecedents.

Keywords: One-stage; Full-mouth Disinfection; Nd:YAG; Gain in Clinical Attachment; Cost-effective Procedure; Thermal Confinement

\section{Introduction}

The aim of today's Periodontal treatment is one of the biggest challenges of today's dental disciplines, which have been developing over the last 40 years. Thanks to rapid evolution, new innova- tions in periodontal diagnosis and therapy, impact of the genomic and proteomic era as well as the advances in cell biology, bacteriology and virology, tremendous progress is achieved. The challenge in periodontology lies not only in searching a cost-effective periodontal therapy for a disease that has an important impact on gen-

Citation: Beatrijs Deruyter. "One-stage, Full-mouth Sulcular Debridement with FRP 1064 Nd:YAG as Adjunctive to a Full Mouth Initial Periodontal Therapy: A Clinical Case Series Retrospective Study". Acta Scientific Dental Sciences 6.1 (2022): 45-55. 
eral health, but also in the impact of the physiological and psychological consequences of the surgical and nonsurgical techniques on patients. Behavioral and economic patient factors have driven the practitioner to look for more acceptable therapies in combination with up-to-date knowledge [1,2].

The ideal therapy should: 1) Be effective for most to all stages of periodontal disease. 2) Be a one-stage, full-mouth procedure to prevent cross infection. 3) At least prevent any further loss of hard or soft tissue. 4) Preferably cause regeneration of hard and soft tissue. The therapy is usually evaluated by the outcomes of probing the attachment levels and radiographic analysis, since re-entry operations to prove true gain of attachment with formation of cementum with insertion of collagen fibers coronal to the previous attachment level are rather contra-productive. Both criteria, probing and radiographic evaluations, have their limitations in accuracy. Probing is as such an estimation of improvement of health of the surrounding soft tissue. Radiographic analysis of healing intrabony defects following regenerative surgery is often documented by measurements of standardized and reproducible radiographs and even now with the extend of CBCT improved but avoided and not a standard procedure for measuring. However this is no proof of newly formed root cementum with inserting collagen fibers. It can be junctional epithelium between newly formed bone and curetted root surface. Furthermore, the ideal therapy should be: 5) As non-invasive a procedure as possible. 6) Accessible for all patients, also medically compromised patients. 7) Simple to perform, amenable in chair side time to be cost effective and to be combined with meticulous home care, which leads to beneficial changes in sub gingival microbial ecology. The concept of meticulous homecare is sometimes challenging when patients are left with oversensitive dentition due to previous periodontal care which can be avoided. 8) Finally, given the lack of plethora of dental practitioners the last few years, together with the fact that the prevalence of periodontal disease in the world population affected by it is about $60-70 \%$ - of whom almost $30 \%$ need regular care - there is a need to be able to delegate a part of the dental care to dental auxiliaries like dental hygienists. Since genetics play a role in periodontitis, it is not only individuals with poor oral hygiene who are susceptible to the disease. Since the periodontal pathogens and the host are the protagonists in the condition, in this context it is important that an adequate removal of the supra- and sub-gingival biofilm and calculus and removal of toxins are prerequisites for periodontal health [3].
To be effective, a periodontal treatment has to reduce the biofilm at manageable levels for the immune system. One-stage, fullmouth (OFM) procedures, has been a landmark introduced Marc De Soete, Prof Quirynen., et al. at Leuven University in 1995. It is quite evident that subgingival organisms are capable of occupying all sorts of niches in the oral cavity and acting as reservoirs. These are associated with negative clinical outcomes if they are not removed. Some pathogens appear to recolonize within 7 days of treatment. In this concept the longer the interval between instrumentation sessions, the greater the likelihood of recolonization of treated sites. It is even obvious that translocation takes place during treatment and provokes an inflammatory response since there is close contact between periodontal pathogens and the rest of the oral cavity and even the bloodstream while executing the protocol of scaling and root planing. The introduction of the OFM protocol with the initiation and completion of instrumentation within $24 \mathrm{~h}$, with adjunctive of antiseptic agents to non-periodontal sites (supra- and sub-gingival) was thereby likely to improve clinical outcomes. However, these additional improvements were only modest and there was only a very limited number of studies available for comparison, thus limiting general conclusions about the clinical benefit of full- mouth disinfection. The duration of the included studies ranged from two to eight months, maximum 71 individuals were included and chair time was between 1 hour and two sessions within 24 hours [4]. Furthermore, in one of the consecutive publications of prof W. Teughels: "One-stage, full-mouth disinfection: fiction or reality" is noticed that patients treated in the Leuven group differed from the other studies in reaction and treatment outcome. In 4 (Leuven group) of the 7 studies on one-stage, full-mouth disinfection were performed in two days. Only in the Leuven group there was an additional improvement (lower proportion of pathogens, fewer anaerobic species, larger reduction in spirochetes and motile organisms, additional gain in clinical attachment level up to 1, 7 for single- and 1.5 for multi-rooted teeth [5]. These four studies were prospective studies designed as 'proof of principle' and patients with only severe periodontitis (pockets $>$ or $\geq 7 \mathrm{~mm}$ ) and a significant amount of supra- and sub gingival plaque and calculus were included. In other words, the probability of cross-contamination was very high. The benefits attributed to the adjunctive applied antiseptics in conjunction with sub gingival debridement are indeed limited in general. Another consecutive study conducted of Prof Quirynen et. al. in which the full mouth approach was applied but without the use of chloorhexidine, showed clearly less benefit $[5]$.

Citation: Beatrijs Deruyter. “One-stage, Full-mouth Sulcular Debridement with FRP 1064 Nd:YAG as Adjunctive to a Full Mouth Initial Periodontal Therapy: A Clinical Case Series Retrospective Study". Acta Scientific Dental Sciences 6.1 (2022): 45-55. 
There were several possible explanations for the reported success of this kind of one-stage, full-mouth disinfection protocol. The observation that patients experiencing an increase in body temperature the evening after the second day of the One-stage, fullmouth treatment showed the most impressive improvement. This suggests that part of the success might be related to an increased immunologic response (e.g. a Schwartzman reaction). Indeed, the second introduction of bacteria/lipopolysaccharides from the sub gingival area into the underlying tissues (during treatment of the remaining quadrants), 24 hours after the first stage of treatment, could have led to a local Schwartzman reaction. This Schwartzman reaction is the result of a bacteremia by repeated contact with periodontopathogens and the bloodstream. This can create problems for medically compromised patients. Through the worldwide standard protocol of treatment, these patients are usually treated under antibiotic prevention protocol. Under these circumstances it is possible that one can create medically compromised patients during the treatment procedure by the nature of the pathogens [6]. In the above-mentioned study, although not designed to investigate the hyperthermic reaction after scaling, it is noteworthy that seven out of 11 patients whose body temperature rose above $37^{\circ} \mathrm{C}$ after the second day, had an overall average pocket depth reduction of $>$ $3.5 \mathrm{~mm}$, whereas this was only the case for four of the remaining 13 patients who did not develop hyperthermia. This effect, after repeated scaling and root planing, has also been suggested in previous publications. In one study, Pawlowski., et al. 2005 left three teeth in one quadrant untreated, while all other teeth were scaled and root planed. The untreated sites showed a significant probing depth reduction and gain in attachment and the number of Treponema denticola and $P$. intermedia species, counted in sub gingival flora of these sites, were reduced for up to 12 weeks. Also, these authors suggested that up to half of the improvements observed following scaling and root planing may be a result of other factors than the removal of plaque, calculus and irritants [4]. In literature, the Leuven studies were often incorrectly quoted or interpreted because in these studies the baseline probing depths and attachment levels were measured immediately after scaling and root planing. Other measurements would be discussed since the patients enrolled in the study, showed significant amounts of supraand sub gingival calculus to the extent that pocket probing prior to scaling would have been unreliable. This complicates comparisons with other clinical trials. However, because this method had been applied in both test and control groups, it cannot contribute to differences between both treatment strategies. One should also take into consideration that the patients did not get any additional periodontal therapy over the entire 8-month period and overall degree of plaque control was sometimes not optimal since Belgium was deprived of oral hygienists. The clinical results in the control group have been considered to be below what one can expect from a thorough mechanical debridement. From an economical point of view this frequent low-recall treatment strategy contributes in the cost-effectiveness of periodontal therapy and to the patients' reliability to follow the program. It is at least reported that in severe periodontitis, where the number of periodontopathogens in saliva is increased significantly, the possibility of translocation and crosscontamination is most likely to be in a horizontal and vertical way. The one-stage, full-mouth approach will result in an immediate reduction of microbial load and in delayed novo plaque formation [8]. The closest to this study is the Koshy study, where a less potent disinfection protocol was used [9]. Nevertheless, this approach resulted in a $26 \%$ increase in pocket site reduction compared to a staged approach. Given the development of resistance to antimicrobial agents, it should be encouraged to look for alternatives or adjunctives in treatment protocol to reduce the use of antibiotics. Considering the number of bacteria knowingly capable of causing serious health threatening conditions at baseline of the treatment, and the risk of bacteremia constantly present during the initial periodontal treatment, it might be wise to consider the use of suitable, suitable, well selected antipathogenics, by preference preference followed by probiotics, not only for medically compromised patients, but to extend it to all cases where the number of bacteria is causing concern for general health, since not all patients are aware of conditions that might lower the immune defense system and each patient is an individual who may not always fit in the average determined by science [10].

In this present study of One-stage, Full-Mouth disinfection of Scaling (FMS) and additional pocket debridement with a Free Running Pulsed (FRP) Nd:YAG laser for Laser Attachment Procedure (LAP), results are compared to the Leuven studies. When indicated by bacterial assessment, systemic antimicrobials are used at the initial therapy, but since the conditions under treatment were at the start all advanced cases of periodontal condition, this is usually the case. Mouth rinses based on chlorhexidine are avoided in this protocol for the following reasons: 1) Since chlorhexidine is 
an irritant. Care should be taken to use it on sensitive tissues. Allergies have been reported with $0.5 \%$ concentrates on skin [11]. 2) Discoloration of teeth associated with the use of chlorhexidine has been extensively described and would only make the protocol less cost-effective. Where the Leuven study took two subsequent chair-time treatments in 24 hours, the Koshy study took a chair time of 1 hour but was less potent in killing pathogens and with less prominent clinical outcome. Changing the disinfection protocol could lead to a more potent treatment plan with even more convincing treatment results in about the same chair time as the Koshy study and with a minimal risk for, and maximal acceptance by the patient and practitioner. Consequently, the periodontal treatment will have to include the removal of supra- and sub gingival hard and soft dental plaque, biofilm and endotoxins which are penetrating the soft tissue, cementum and, last but not least, dentine tubules which are considered serious reservoirs for bacterial load [12]. The estimation of density per square $\mathrm{mm}$ dentine tubules is presumed to be 30 000-60 000 per $\mathrm{mm}^{2}$. This difference in density of dentine tubules is the main factor determining whether the patient will be suffering from hypersensitivity already before and certainly after traditional periodontal treatment. The loss of attachment causes the exposure of unprotected dentine. Furthermore, the inflammatory response on the traditional treatment, open or closed procedures, causes even more loss of tissue, exposing more naked dentine [13]. The research about species and quantity of biological load in dentine tubules has been more extensively studied in endodontic research literature. The traditional approach to eliminate these reservoirs is root planing. This disinfection protocol effectuated by scraping with hand or rotary instruments the layer of infected cementum and dentine, where the biofilm can penetrate at least more than $1000 \mu \mathrm{m}$ in dentine, are not only provoking a tremendous discomfort for the patient, but also ruled out for frequent repetition. Each treatment causes loss of dental material, sometimes inducing endodontic problems. This will reduce the regeneration possibilities by removing the Enamel Remnants of Malassez, remnants of the root formation, capable of producing Enamel Matrix Proteins(EMP), signaling molecules for regeneration [14]. Since a periodontal patient is genetically predestinated, repetitive treatment plans are consequently imposed. The hypersensitivity caused by the periodontal process itself and even more by traditional treatment protocol is a serious concern. In a comprehensive questionnaire survey, severe painful symptoms were reported in $26 \%$ of the subjects 6 months to 5 years after completion of treatment, while $16 \%$ treated non-surgically reported pain symptoms.
These patients suffering from severe root dentine hypersensitivity have need for urgent treatment, however the present conventional methods available provide an unpredictable outcome and usually give only temporary relief [15]. Removal of the infected cementum causes not only more hypersensitivity, however, it also causes more loss of dentine tissues, opening more dentine tubules, creating more reservoirs for pathogens and regeneration is even more compromised. In the end, because of this hypersensitivity the patient is less motivated or even unable to keep up a strict home dental and periodontal care plan. From the practitioner's experience, this issues can be avoided by changing the protocol.

\section{Photothermal approach}

Lasers are important to meet the criteria for periodontal treatment. The first laser was assembled by Theodore Maiman in 1960. The principle mostly relies on the concept that every kind of photonic energy (wavelength) is interacting with specific photo-acceptors and thereby transformed in energy. The most frequent use of this mechanism of photonic energy conversion in laser medicine is thermal effect, in other words, heating. By different dosage of energy, one can deliver different effects. Very short pulsed lasers like Free Running Pulsed Lasers (FRP), play an important role in therapeutic applications. The FRP laser generates high peak powers (1000W and more are possible). This energy is spread into the tissue and is characterized by a thermal time constant for each tissue, called thermal relaxation time. With short pulsed lasers, the pulse duration range is considerably less $(50-300 \mu \mathrm{sec})$ than the needed thermal relaxation time, thereby, local high energy delivery does not necessary raise the temperature in the surrounding tissue and cold ablation is possible Thermal relaxation properties enables to work with no or very little anesthesia. This means that the temperatures, once beyond the location of application, are dissipated and do not exceed $45^{\circ} \mathrm{C}$. At this temperature, the patient does not suffer from the temperature rise, since the action takes place within physiological temperature ranges without surpassing the patient's pain threshold for heat. This is also a safety measure to avoid thermal damage. Since thermal relaxation is sufficiently available, carbonization will not occur in this protocol and has always to be avoided since it delays healing [16]. The rate, expressedin $\mathrm{Hz}$ at which energy(from a pulsed laser) is delivered is important as well. Nowadays lasers with even nano, pico and femto seconds laser pulses are on the market, ensuring even higher peak powers and even more thermal relaxation. The closer to the energy source, the higher the temperature and by dissipation of the energy 
absorbed by the tissue, the temperature will drop. The result of these photonic interactions at different temperatures on dental structures induces at different energy levels : spallation, vitrification and carbonization, vaporization, dehydration-ablation, coagulation by irreversible protein denaturation, reversible protein interaction, hyperthermia without protein alteration, the latter also called biomodulation [17]. For the Nd:YAG, used in this study, settings of $1 \mathrm{~W}, 10 \mathrm{~Hz}, 100 \mathrm{~mJ}$ per pulse and a $126 \mu \mathrm{sec}$ pulse width, Peak powers of 793,6 W per pulse and temperatures of more than $1000^{\circ} \mathrm{C}$ are reached. The thermal profile between $1100-250^{\circ} \mathrm{C}$ is of practical use in the protocol for treating periodontal disease by surely destroying pathogens (bacterial and viral) and its toxins but also resolving the challenge in search for an adequate solutions for dentin tubules apertures, causing hypersensitivity and being reservoirs for pathogens and toxic load up to $1000 \mu \mathrm{m}$. These temperatures will restructure the dentine, vaporize all proteins and H2O and there is contraction of axis lattice of hydroxyapatite This avoids the need for root planning, which leaves the patient with sensitivity, loosing hard dental structure and leaving an excludible open dentine tubules, susceptible to reinvasion by a new biofilm. This sensitivity compromises inevitable strict dental home care, crucial for periodontal maintenance [18-20]. The temperature profile between $250-100^{\circ} \mathrm{C}$ is responsible for vaporization, ablation and is the most commonly used thermal effect of lasers in the surgical field. With appropriate settings, the granulation tissue of the infected pocket lining will be ablated, creating a fresh bleeding. Healthy tissue would need more energy to be removed and under the used settings, is preserved, avoiding recessions. From $90-70^{\circ} \mathrm{C}$ coagulated protein will cause hemostasis with a fresh created blood cloth. From $65-55^{\circ} \mathrm{C}$ irreversible protein denaturation in absence of pretreatment. Between $54-44^{\circ} \mathrm{C}$ bio-inhibition and reversible damage will occur. As a result of the cellular repair mechanism, some temperature rise is used for reversible damage in regeneration procedures [21]. Finally, the temperature dissipates to 43$37^{\circ} \mathrm{C}$, comparable to fever and in the range of bio-stimulation and preconditioning under hyperthermia conditions. This Hyperthermia is interesting in the therapeutic field and partly responsible for laser effects with consequences on immune and regenerative response level. Here Heat Shock Proteins (HSP) play an important role. This class of proteins are considered molecular chaperones. Chaperones help newly synthesized or improperly folded proteins to form into their most stable, native structure. Most mammalian cell lines respond to a temperature increase of at least $5-6^{\circ} \mathrm{C}$, which is at $42^{\circ} \mathrm{C}$ if the optimal growth temperature of the cell is at $37^{\circ} \mathrm{C}$. HSP are found in virtually all organisms, they consist of several kinds of proteins, named by their molecular weight. HSP70 is thereby one of the most widespread HSP. It accounts for 5\%-10\% of the total protein content of a cell and is upregulated to $15 \%$ in the presence of cellular insults. These insults include heat stress, oxidative stress, anoxia, cytokines, viral infection and heavy metals. HSP70 are also thought to be involved in response to conditions such as inflammation, cancer, atherosclerosis, and such amyloid diseases as Alzheimer's [22]. Pretreatment of cells and tissue before exposing it to cellular insult, even high temperatures such as with ablation/coagulation or any other form of tissue damage by trauma can minimize the damage on a macroscopic scale to the collateral structures. Photo-biomodulation, known since the sixties and already investigated in the early eighties, are better understood by the work of Mester and especially T. Karu. We remember that discrete energy levels of photonic energy, affects the mitochondria of the cell, primarily cytochrome-C oxidase, containing heme and copper centers which absorb red and near-infrared light in the electron transfer chain and porphyrins on the cell membrane [23-25]. One should note that light therapy methods based on photochemical conversion of photo-absorbing molecules are not laserspecific. Conventional sources generating the appropriate wavelength can also be used (LED), but lasers are more practical sources with the advantages of being monochromaticity (very specific in wavelength), coherent (unique for lasers and enabling more efficacy) and collimation (easy to apply in a concentrated way). It is the coherency effects that cause the laser light speckles (very small heat spots) which can cause local heating of inhomogeneous tissue when laser light strikes different types of molecules and cells causing interaction. Bear in mind that in light/cell interaction at $1 \mathrm{~J} / \mathrm{cm}^{2}$ is sufficient to start off chemical processes and can be used as prevention level of irradiation, where 2, 3 and 4 Joules are curative levels according to the seriousness of the lesion and in vitro saturation is reached at $4 \mathrm{~J} / \mathrm{cm}^{2}$. Slightly different figures can be found in different sources. Depending on the administered energy different types of chemical reactions take place and different outcomes can be expected. The effect of this discrete administration of monochromatic visible and near infrared radiation on cells with its primary and secondary effect has already been described by Arvanitaki and Chalazonitis in 1947, where it is advanced that 
mitochondria of mammalian cells have photo-sensitivity [26]. Lately the importance of cytochrome c- cardiolipin complex and its redox potential in apoptosis, has been described $[27,28]$. In summary from the pioneers' conclusions, their expectations from biomodulation are: 1) Antalgic effects by increase of production of endorphins [29]. 2) Gate-control by hyperpolarization of the cellular membrane. 3) Euthrophic effect (reconstruction of soft and osseous tissue starting from the adult matrix cells) since production of growth factors like Platelet Derived Growth factor, Fibroblast Gf and Transforming Gf take place at biomodulation temperatures. However different cell lineages will respond different to this kind of energy levels [30-32]. Typical wavelengths used for biomodulation are in the range of visible and near infrared wavelengths from $450 \mathrm{~nm}-+/-1500 \mathrm{~nm}$ for their absorption properties in light sensitive chromophores from the tissue. One wavelength to the other can differ in penetration depth according to the absorption coefficient caused by the specific chromophores: this is called the optical window and dictates the absorption curves of the wavelengths. The absorption coefficient is expressed by Lambert-Beer's law where $A=I_{0}-I / I_{0}$, where A stands for the absorption coefficient and I for intensity of radiation measured at a standard level in comparison with intensity at baseline. It is necessary to bear in mind that $1064 \mathrm{~nm}$ is also an invisible near-infrared wavelength with negligible absorption in water and thus penetrating deeply into the tissue. Besides the use of energy for tissue destruction, energy distribution will eventually result in distribution of the energy in a threedimensional way into the tissue. The latter type of low-level laser administration of energy will always occur automatically next to high-energy administration by dissipation of the energy [17]. We can call it also thermotherapy or biomodulation on distance [21]. Discrete temperature changes in ranges from fever $\left(>37^{\circ} \mathrm{C}\right) \mathrm{ob}-$ served by Prof. Quirynen., et al. seem to have a beneficial effect on the immune response, regeneration and activation of tissue in periodontal disease.

\section{Materials and Methods}

Fifty-three patients, all Caucasian (aged 20 to 69, mean age 50 ) were included in this relatively small retrospective study in a private practice. In an attempt to make the samples as homogenous as possible, all patients were selected on having a serious periodontal condition comparable to the studies of Prof Quirynen., et al. All patients had the same kind of assessment and treatment protocol. The following parameters were included: age, sex, DPSI (Dutch Periodontal Screening Index) which are replaced by now by Generalized, stage III and IV, pocket depth of most significant pockets, Bacteriological assessment of the most significant pockets. When indicated, antibiotic medication was prescribed only at initial treatment in accordance to the bacteriological test results containing counts and specimen, dentition in upper and lower jaw to evaluate dentition loss in long term results, previous periodontal program or not, smoker/non-smoker. Date of the start of the program to evaluate some long-term results and assess the value of the treatment. From the 53 patients 34 patients were female, 19 male. 17 patients were smokers, 36 were nonsmoking. All patients following the program were assessed and under regular surveillance (some for up to 10 years). The same procedure was followed for a couple of hundred patients for 30 years, but data were not always gathered in the exact same way and thereby excluded. Other excluding factors were slight differences in protocol, since over the years the protocol was adapted to make the procedure as cost-effective as possible. Slight modifications were made during the 30-year time span to make the procedure as cost-effective and patient-friendly as possible, taking into account that data and controllability were important as well.

\section{Preliminary procedure}

At the intake, the patients are assessed for general health condition by a list of questions in the anamnesis All patients were checked for oral hygiene before and after the initial treatment and were having the same treatment protocol, in other words the patients were sharing the same kind of diagnostic protocol, treated for at least a periodontal condition with loss of hard and soft tissue,. Periodontal parameters were registered by measuring pocket depth, using the PerioWise Probes; dental plaque registered; bleeding on probing; recessions measured in mm; mobility assessed, having a serious plaque- and bleeding index with at least a periodontal condition DPSI 3+ (pockets $>4 \mathrm{~mm}$ bleeding on probing and loss of hard and soft tissue) up to DPSI 4 (pockets $>5 \mathrm{~mm}$, bleeding on probing,) loss of hard and soft tissue and mobility at the beginning of the program and risk of losing dentition. Four samples for bacteriological assessment are taken with paper-points at the most significant pockets. The bacteriological assessment by dark field microscope and Gram staining can be performed if urgent for choice of antibiotics and was done in case of severe periodontal abscess. For bacterial assessment and counts by DNA (PCR.), four of the most significant pockets were chosen for taking samples with sterile paper points after leaving it to absorb the crevicular fluid for about $10 \mathrm{sec}$ and taken into a sterile container. The diagnostic samples were assessed by ADD (Advanced Dental Diagnosis, Malden, Netherlands) before treatment. The same pockets were revisited after eight weeks, and again six months later (in other words, eight months 
after treatment) controllable by X-Ray, taken in the same position. Dental X-ray of the full dentition is taken by panoramic X-ray, for maximum of information by minimum of radiation. This X-ray is assessed for bone loss, eventual dental decay, inadequate fillings causing periodontal issues and other information that might be important for diagnosis, assessment and evaluate compromising situations for treatment outcome. Other observations like apical complications by endodontic involvement, periodontal abscesses, retained elements and other abnormalities are taken in consideration. Further on, the patient has a new assessment on periodontal status about every six months, since all patients are chronic periodontal cases. Only at the initial treatment antibiotics were used according to the bacteriological results, giving bacterial counts and species of pathogens present. 17 patients received traditional treatment prior to the protocol with laser, eight of them with open procedures. Two patients had up to 4 open flap procedures over 7 years, with loss of dentition and poor result. One patient had been on an oral hygienist program of scaling and root planing, four times a year, during several years in a neighboring country has been performed the last ten years. It is from this pool of patients, all with the same kind of assessment, that the study was taken.

\section{Previous comparable studies}

The most effective results were obtained by the studies of Prof. Quirynen., et al. Like in the present study, the procedure emphasizes the importance of one-stage, full-mouth procedure and, although this was not the aim of the study, it is observed that thermal effect has an additional impact on the improvement outcome of the treatment. Besides the group of Prof Quirynen, only the Koshy., et al. (2005) study really presents a full-mouth disinfection in order to reduce bacteria from other oral niches, was performed in one hour of chair time, using a weak antiseptic disinfection protocol. This study shows comparable results to the study of Prof. Quirynen (2006) but lacks in thermal influence on results with remarkable differences, although their outcome still shows an improvement of treatment results in reduction of periodontal index.

The experimental design of the Leuven group contained five separate treatment strategies for a total of 71 patients:

- $\quad$ A NC $(n=15)$ group where scaling and root planing were performed quadrant by quadrant as traditional treatment, starting with first quadrant, followed at 2- week interval by second, third and fourth quadrant; thus six weeks between first and last quadrant without adjunctive products.
- A control group FRp ( $\mathrm{n}=14)$ full mouth root planing in two consecutive days without the use of adjunctive products.

- $\quad$ One of the three positive control groups FMCHX ( $(n=14)$ : including a one-stage, full-mouth disinfection (OSFMD) (Quirynen., et al. 1995) followed by the use antiseptic mouth rinse chlorhexidine $0.2 \%$ (Corsodyl, Glaxo-SmithKline, Genval, Belgium) for two months.

- $\quad$ Another positive group FMF $(n=14)$ OSFM with AmF/ $\mathrm{SnF}_{2}$ (Meridol mouthrinse, GABA International, Münchenstein, Switserland) for two months.

- A last group ( $\mathrm{n}=14) \mathrm{FMCHX}+\mathrm{F}$ where the OSFM is combined with chlorhexidine $0,2 \%$ for two months, followed by $\mathrm{AmF} / \mathrm{SnF}_{2}$ for the following six months.

For all groups, except the NC group, the scaling and root planing was performed and completed within $24 \mathrm{~h}$ (starting with the lower jaw). Scaling and root planing was performed under local anesthesia using periodontal hand instruments (Gracey curettes, $\mathrm{Hu}$ Friedry, Chicago IL, USA). For the three positive control groups, the mechanical debridement was combined with an extensive chairside chlorhexidine application. In sum, after completion of the two first quadrants, and at the 1-week follow-up visit, all pockets were irrigated with a $1 \%$ chlorhexidine gel (three times repeated in 10 minutes). The patients also received standard oral hygiene instructions immediately after the first session of scaling and root planing.

Procedure Full Mouth Scaling-Laser Attachment Procedure (FMS:-LAP). All appropriate safety measures are to be taken when lasers are used: Secured room is used with correct laser warning signs for lasers of Class 4 posted outside the door. Note that the use of electrosurgical devices, high-speed rotary instruments and ultrasonic devices create hazards as well and have to be taken in consideration (LIA Laser Institute of America ANZI 136.3 2011).

\section{Informed consent}

Consent was given by the fully (technically and financially) informed patient and retained in the digital treatment notes.

Laser operating parameters: FOTONA Fidelis + (Ljubljana, Slovenia); FRP Nd:YAG 1064nm near infrared wavelength absorbed by dark pigments and hemoglobin; Class 4 laser; NOHD : 25,3m; Free Running Pulsed VSP: 126 s pulse width; Fiber delivery system: $300 \mu \mathrm{m}$ spot diameter quartz fiber ;Aiming beam $632.8 \mathrm{~nm}$ diode of $1 \mathrm{~mW}$; Max Output: 15W; Average power setting of 1W; Pulse 
Frequency: $10 \mathrm{~Hz}$; Energy per pulse: 100mj; Peak Power: 793,6W per pulse.

\section{Treatment delivery sequence}

The procedure was done under magnification with loupes (HEINE HR 2.5) over safely goggles. Anesthetic was only administered in small amounts on sites of oversensitivity for scaling if needed (Articainehydrochloride 72,00mg-Epinefrinetartrate $16,38 \mathrm{mcg}$ administered with needle (30G-0,30x21) (about 0,3cc per site).

Full-mouth scaling supra and sub gingival was performed to remove hard and soft dental plaque and biofilm as much as possible,. The Nd:YAG hand piece was assembled and test fired on dark articulation paper with low settings. The bare fiber extends several $\mathrm{mm}$ out of the cannula along the maximum pocket depth in order to avoid contact of cannula with the tissue or enabling blood to be aspired by the silicon jacket or cladding of the fiber. All pockets were treated with the same technique. Used in contact with soft and hard tissue in the pocket, using sweeping pencil technique, wiping the pocket, creating fresh bleeding by removing the infected lining of the pocket by ablation. If no bleeding occurs the pocket is disinfected for max 30" per pocket, using the same pencil technique with the aim of disinfection of hard and soft tissue and closing dentine tubule openings, to reduce bacterial reservoirs. After laser treatment the teeth were polished with $0.4 \%$ sodium fluoride paste (Detartrine Fluoré, Septodont) and patient could rinse with Listerine in water. The whole procedure takes on average one hour to complete (scaling, laser treatment, polishing). Treatment records and settings are listed in patient's digital records. Post-operative instructions: The following post-operative instructions were given to the patients: 1) Normal dental care and rinse with 3\% hydroperoxide in water twice a week. 2) Antibacterial medication if indicated by the test. 3) Refrain from smoking for smokers. 4)Probiotics are advised to be taken from the start of the antibacterial medication intake. 5) Refrain from interdental brushes since attachment is negatively influenced by the pressure of the metal wire in the brush 6) Contact the office if complications should occur, which is not expected as such. Patients were assessed for periodontal index and results of attachment gain two months after the full-mouth scaling combined with Laser Attachment Procedure (LAP) in order to evaluate the progress in attachment gain. The next appointment is six months later, which is at 8 months from baseline. Follow-up is another six months later, at 14 months from first procedure. Usually, slight progress is still achieved in attachment gain and the results can be maintained by retreatment after 14 months but this time without any antibacterial agents like antibiotics, just strict dental home care like instructed after the procedure. In the study, moderate pockets up to $5.5 \mathrm{~mm}$ were separated from deep pockets to evaluate if differences occur. Results: Almost all studies performed until present differ in treatment protocol. As such, statistics have to be evaluated with caution. In analogy to the other studies, two groups have been created: the first group with medium pockets (up to 5-mm pocket depth) and a second group with deep pockets (over $5-\mathrm{mm}$ pocket depth). During the test, only the initial treatment has been taken in consideration to be able to compare with the other studies.

Table 1 below provides an overview of pocket probing depths throughout the different procedures.

\begin{tabular}{|l|c|c|c|c|c|c|}
\hline \multirow{2}{*}{ Strategy } & \multicolumn{5}{|c|}{ Pocket probing depth (mean and SD) } \\
\hline & \multicolumn{3}{|c|}{ Medium pockets } & \multicolumn{3}{c|}{ Deep pockets } \\
\hline & Month 0 & Month 2 & Month 8 & Month 0 & Month 2 & Month 8 \\
\hline FMS+LAP+IAB & $4,3 \pm 0,7$ & $1,6 \pm 0,8$ & $1,3 \pm 0,8$ & $7,0 \pm 1,2$ & $2,8 \pm 1,1$ & $1,9 \pm 1,0$ \\
Single rooted & & & & & & \\
NC & $4,8 \pm 0,2$ & $3,5 \pm 0,4$ & $3,5 \pm 0,5$ & $7,2 \pm 0,5$ & $5,0 \pm 0,9$ & $4,9 \pm 0,9$ \\
Frp & $4,9 \pm 0,3$ & $3,5 \pm 0,6$ & $3,5 \pm 0,5$ & $6,8 \pm 0,3$ & $4,4 \pm 0,7$ & $4,3 \pm 0,8$ \\
FMCHX & $4,9 \pm 0,2$ & $3,2 \pm 0,4$ & $3,1 \pm 0,4$ & $6,9 \pm 0,5$ & $4,3 \pm 0,8$ & $4,3 \pm 0,7$ \\
FMF & $4,7 \pm 0,2$ & $3,3 \pm 0,3$ & $3,3 \pm 0,4$ & $6,9 \pm 0,6$ & $4,5 \pm 0,8$ & $4,5 \pm 0,7$ \\
FMCHX + F & $4,9 \pm 0,2$ & $3,3 \pm 0,5$ & $3,2 \pm 0,4$ & $7,1 \pm 0,6$ & $4,4 \pm 0,9$ & $4,3 \pm 0,9$ \\
Multi-rooted & $4,7 \pm 0,2$ & $3,8 \pm 0,5$ & $3,7 \pm 0,6$ & $7,3 \pm 0,7$ & $5,1 \pm 0,9$ & $5,0 \pm 0,9$ \\
NC & & & & & & \\
Frp & $5,2 \pm 0,2$ & $3,8 \pm 0,4$ & $3,7 \pm 0,4$ & $7,3 \pm 0,5$ & $4,7 \pm 0,8$ & $4,7 \pm 0,7$ \\
FMCHX & $4,9 \pm 0,4$ & $3,3 \pm 0,5$ & $3,4 \pm 0,7$ & $7,3 \pm 0,6$ & $4,5 \pm 0,9$ & $4,6 \pm 0,7$ \\
FMF & $4,7 \pm 0,3$ & $3,5 \pm 0,5$ & $3,3 \pm 0,4$ & $7,0 \pm 0,9$ & $4,8 \pm 1,0$ & $4,7 \pm 1,0$ \\
FMCHX + F & $4,6 \pm 0,3$ & $2,9 \pm 0,6$ & $3,0 \pm 0,7$ & $7,5 \pm 0,7$ & $4,5 \pm 0,8$ & $4,4 \pm 0,9$ \\
\hline
\end{tabular}

Table 1: Pocket probing depths throughout the different procedures. 


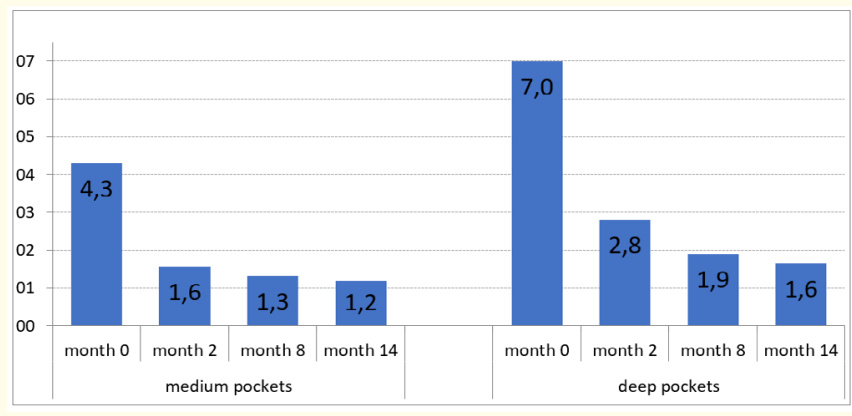

Figure 1: Evolution of pocket depth -FMS+LAP+IAB over time.

The graph (Figure 1) shows the pocket depth reduction for the two groups over time lapses similar to those suggested by other studies on traditional quadrant treatment and one-stage, fullmouth procedures, with or without an additional disinfectant.

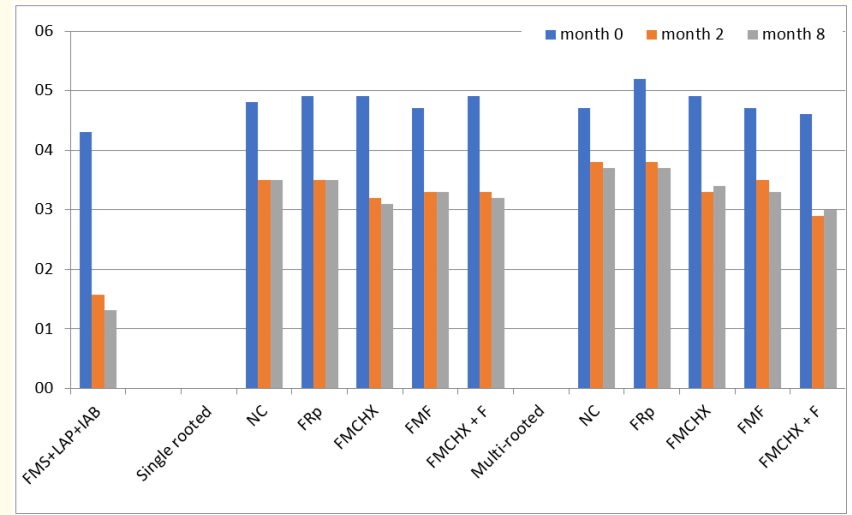

Figure 2: Evolution of pocket depth: medium pockets.

The next graph (Figure 2) shows the pocket depth reduction over time in comparison to the other studies of traditional quadrant treatment and one-stage, full-mouth procedures for medium pocket depth.

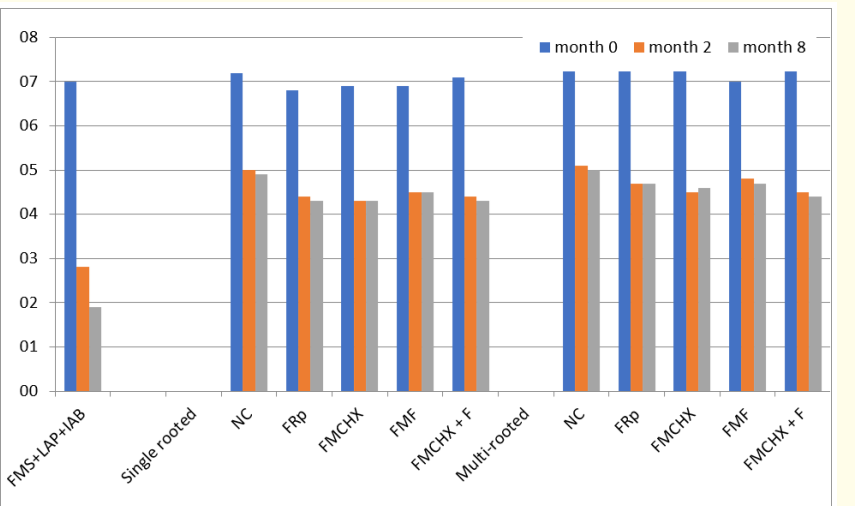

Figure 3: Evolution of pocket depth: deep pockets.
Finally, figure 3 shows the pocket depth reduction over time for deep pockets in comparison to other studies on deep pockets.

T-tests were performed to compare the baseline with the results after 2 months, after 8 months and after 14 months. For all time intervals, pocket depth reduction is found to be significant ( $p$ $<.05$ ). Table 2 provides an overview of the results of the statistical tests.

\begin{tabular}{|c|c|c|}
\hline $\begin{array}{l}\text { Comparison } \\
\text { baseline-2 months }\end{array}$ & $\begin{array}{c}\text { Comparison } \\
\text { baseline-8 months }\end{array}$ & $\begin{array}{c}\text { Comparison } \\
\text { baseline-14 months }\end{array}$ \\
\hline $\begin{array}{l}\text { Mean baseline: } 5.07 \\
\text { Mean } 2 \text { months: } 1.92\end{array}$ & $\begin{array}{l}\text { Mean baseline: } 5.07 \\
\text { Mean } 8 \text { months: } 1.47\end{array}$ & \begin{tabular}{|c|} 
Mean baseline: 5.09 \\
Mean 14 months: \\
1.32
\end{tabular} \\
\hline $\begin{array}{l}\text { Two-sample T (377): } \\
25.14 \\
p=6,88 \mathrm{E}-83\end{array}$ & $\begin{array}{c}\text { Two-sample } \mathrm{T}(346): \\
30.01 \\
\mathrm{p}=1,26 \mathrm{E}-98\end{array}$ & $\begin{array}{c}\text { Two-sample T (314): } \\
31.31 \\
\mathrm{p}=6,71 \mathrm{E}-99\end{array}$ \\
\hline
\end{tabular}

Table 2: T-test results: pocket depth evolution over time.

Results show a statistically significant difference between baseline values and assessment over time. Furthermore, there is a significant difference with other strategies of periodontal management. Of course our sample is more heterogeneous than those of the other studies as the standard deviation is larger from the beginning and remains so. It is questionable, though, whether this has an impact on the results. Finally, it must be said that even without any further or additional treatment during the time of assessment, pocket depths continue to reduce.

Summary of the Finding, Questions and Leads for Further Research

Pocket depth reduction in this study is significant. Lack of losing teeth could be investigated but is not relevant in this study, since the other studies did not take that parameter into account.. The results of this small-scale study are compared to the best possible results achieved by one-stage full mouth procedures by the Leuven group. Although the protocol is different from the Leuven group, and thus caution has to be taken to compare statistics, results are consistently repeatable (30 years), and this 'proof of principle' is the main point of the author. In search of a cost-effective procedure for periodontal management, this protocol is certainly worthwhile to be investigated more closely, even better on multicenter level.

Reattachment of the periodontal ligament and regeneration of hard and soft tissue by stimulation with laser of stem cells in the blood cloth and on the cementum are comparable to the use of PRF and Enamel Matrix Protein (EMP) and combination of the techniques could open even more treatment options [33-35]. Stimulation of fibroblasts and blood supply by bio-modulation effects of lasers are also important for stimulation of regeneration. These 
phenomena have been studied in the early eighties, but should be re-examined in the scope of present state-of-the-art knowledge of biomedicine (genomic and proteomic).

The production of Heat Shock Proteins, necessary to refold the protein structures, the change in redox potential, and production of the protein transcription factors helps in repair and regeneration of damaged cells by any kind of trauma, whether by inflammatory processes, physical and chemical trauma or heat, deserves closer investigation in view of the appropriate laser use in any kind of surgical or non-surgical procedure. Periodontal balance, depending on the correct balance in redox potential could best be managed before implant surgery and contributes to the prevention of peri-implantitis [36]. Most questions might be investigated and discussed with the Biomedical and Bacteriological department. Viral assessment is not taken into account in this study although it should be taken in consideration in future investigations since studies about the influence of Herpes Simplex, Epstein Bar and lately (+)ssRNA viruses on periodontal destruction have concluded that damage is more prominent when certain viruses are present [37]. To my knowledge, with the exception of Dr Maratelli's large scale study from 2015, no one-stage, full-mouth strategy in combination of FRP Nd:YAG has been included in other studies involving laser. Split mouth studies are not reliable because of the abscopal response of laser irradiation. Better knowledge about different laser characteristics is required to compare studies.

\section{Disclosure}

No funding has been involved in this study.

\section{Bibliography}

1. Botelho J., et al. "Economic burden of periodontitis in the United States and Europe - an updated estimation". Journal of Periodontology (2021).

2. Fischer RG., et al. "Periodontal disease and its impact on general health in Latin America. Section V: Treatment of periodontitis". Brazilian Oral Research 34.11 (2020): e026.

3. Chapple IL. "Periodontal diagnosis and treatment--where does the future lie?" Periodontology 51 (2009): 9-24.

4. De Soete M., et al. "One-stage full-mouth disinfection. Longterm microbiological results analyzed by checkerboard DNADNA hybridization". Journal of Periodontology 72.3 (2001): 374-382.
5. Teughels W., et al. "One-stage, full-mouth disinfection: fiction or reality?” Periodontology 50 (2009): 39-51.

6. Kinoshita M., et al. "Immune Mechanisms Underlying Susceptibility to Endotoxin Shock in Aged Hosts: Implication in AgeAugmented Generalized Shwartzman Reaction". International Journal of Molecular Sciences 20.13 (2019).

7. Pawlowski AP., et al. "Clinical effects of scaling and root planing on untreated teeth". Journal of Clinical Periodontology 32.1 (2005): 21-28.

8. Mongardini C., et al. "One stage full- versus partial-mouth disinfection in the treatment of chronic adult or generalized early-onset periodontitis. I. Long-term clinical observations". Journal of Periodontology 70.6 (1999): 632-645.

9. Koshy G., et al. "Effects of single-visit full-mouth ultrasonic debridement versus quadrant-wise ultrasonic debridement". Journal of Clinical Periodontology 32.7 (2015): 734-743.

10. Lamster IB and Pagan M. "Periodontal disease and the metabolic syndrome". International Dental Journal 67 (2017): 6777.

11. Chiewchalermsri C., et al. "Chlorhexidine Allergy: Current Challenges and Future Prospects". Journal of Asthma and Allergy 13 (2020): 127-133.

12. Bosshardt DD and Lang NP. "The junctional epithelium: from health to disease". Journal of Dental Research 84.1 (2005): 9-20.

13. West NX., et al. "Dentin hypersensitivity: pain mechanisms and aetiology of exposed cervical dentin". Clinical Oral Investigation 17.1 (2013): S9-19.

14. Bozbay E., et al. "Preservation of root cementum: a comparative evaluation of power-driven versus hand instruments". International Journal of Dental Hygiene 16.2 (2018): 202-209.

15. Farmakis ET., et al. "The influence of different power settings of Nd:YAG laser irradiation, bioglass and combination to the occlusion of dentinal tubules". Photomedicine, and Laser Surgery 31.2 (2013): 54-58.

16. Bornstein ES. "Why wavelength and delivery systems are the most important factors in using a dental hard-tissue laser: a literature review". Compendium of Continuing Education in Dentistry 24.11 (2003): 837-8, 41, 43 passim; quiz 48. 
17. Lukac N., et al. "Ablation characteristics of quantum square pulse mode dental erbium laser". Journal of Biomedical Optics 21.1 (2016): 15012.

18. De Magalhaes MF., et al. "A morphological in vitro study of the effects of Nd:YAG laser on irradiated cervical dentin". Photomedicine, and Laser Surgery 22.6 (2004): 527-532.

19. Klinke T., et al. "Antibacterial effects of Nd:YAG laser irradiation within root canal dentin". Journal of Clinical Laser Medicine and Surgery 15.1 (1997): 29-31.

20. Kim K., et al. "High-intensity Nd:YAG laser accelerates bone regeneration in calvarial defect models". Journal of Tissue Engineering and Regenerative Medicine 9.8 (2015): 943-951.

21. Lukac M., et al. "Variable heat shock response model for medical laser procedures". Lasers in Medical Science 34.6 (2019): 1147-1158.

22. Westerheide SD., et al. "HSF transcription factor family, heat shock response, and protein intrinsic disorder". Current Protein and Peptide Science 13.1 (2012): 86-103.

23. Tafur J and Mills PJ. "Low-intensity light therapy: exploring the role of redox mechanisms". Photomedicine, and Laser Surgery 26.4 (2008): 323-328.

24. Erusalimsky JD and Moncada S. "Nitric oxide and mitochondrial signaling: from physiology to pathophysiology". Arteriosclerosis, Thrombosis, and Vascular Biology 27.12 (2007): 2524-2531.

25. Chen AC., et al. "Low-level laser therapy activates NF-kB via generation of reactive oxygen species in mouse embryonic fibroblasts". PLoS One 6.7 (2011): e22453.

26. Fernandes AB., et al. "Photobiomodulation: Shining Light on COVID-19". Photobiomodulation, Photomedicine, and Laser Surgery 38.7 (2020): 395-397.

27. Hough MA., et al. "NO binding to the proapoptotic cytochrome c-cardiolipin complex". Vitamins and Hormones 96 (2014): 193-209.

28. Albuquerque-Pontes GM., et al. "Effect of pre-irradiation with different doses, wavelengths, and application intervals of lowlevel laser therapy on cytochrome c oxidase activity in intact skeletal muscle of rats". Lasers in Medical Science 30.1 (2015): 59-66.

29. Chellappa D and Thirupathy M. "Comparative efficacy of lowLevel laser and TENS in the symptomatic relief of temporomandibular joint disorders: A randomized clinical trial". Indian Journal of Dental Research 31.1 (2020): 42-47.
30. Engel KW., et al. "Cell lineage responses to photobiomodulation therapy". Journal of Biophotonics 911.12 (2016): 11481156 .

31. Usumez A., et al. "Effects of laser irradiation at different wavelengths $(660,810,980$, and $1,064 \mathrm{~nm})$ on mucositis in an animal model of wound healing". Lasers in Medical Science 29.6 (2014): 1807-1813.

32. Aykol G., et al. "The effect of low-level laser therapy as an adjunct to non-surgical periodontal treatment". Journal of Periodontology 82.3 (2011): 481-488.

33. Tenore G., et al. "Management of Medication-Related Osteonecrosis of the Jaw (MRONJ) Using Leukocyte- and PlateletRich Fibrin (L-PRF) and Photobiomodulation: A Retrospective Study". Journal of Clinical Medicine 9.11 (2020).

34. Miron RJ., et al. "Use of platelet-rich fibrin in regenerative dentistry: a systematic review". Clinical Oral Investigation 21.6 (2017): 1913-1927.

35. Miron RJ., et al. "Twenty years of enamel matrix derivative: the past, the present and the future". Journal of Clinical Periodontology 43.8 (2016): 668-683.

36. Chapple IL. "Reactive oxygen species and antioxidants in inflammatory diseases". Journal of Clinical Periodontology 24.5 (1997): 287-296.

37. Martelli FS., et al. "Efficacy of the ND: YAG laser therapy on EBV and HSV1 contamination in periodontal pockets". Igiene e Sanita Pubblica 71.4 (2015): 369-385.

\section{Assets from publication with us}

- Prompt Acknowledgement after receiving the article

- Thorough Double blinded peer review

- Rapid Publication

- Issue of Publication Certificate

- High visibility of your Published work

Website: www.actascientific.com/

Submit Article: www.actascientific.com/submission.php Email us: editor@actascientific.com

Contact us: +919182824667 